\title{
Subordination and Superordination Results for Normalized Analytic Functions Associated with Frasin Operator
}

\author{
Abbas Kareem Wanas ${ }^{1}$ and Sibel Yalçin ${ }^{2}$ \\ ${ }^{1}$ Department of Mathematics, College of Science, University of Al-Qadisiyah, Diwaniya, Iraq \\ e-mail: abbas.kareem.w@qu.edu.iq \\ ${ }^{2}$ Department of Mathematics, Faculty of Arts and Science, Uludag University, Bursa, Turkey \\ e-mail: syalcin@uludag.edu.tr
}

\begin{abstract}
In this paper, we derive some applications of first order differential subordination and superordination results involving Frasin operator for analytic functions in the open unit disk. Also by these results, we obtain sandwich results. Our results extend corresponding previously known results.
\end{abstract}

\section{Introduction}

Let $H=H(U)$ indicate the class of analytic functions in the open unit disk $U=\{z \in \mathbb{C}:|z|<1\}$ and let $H[a, n]$ be the subclass of $H$ consisting of functions of the form:

$$
f(z)=a+a_{n} z^{n}+a_{n+1} z^{n+1}+\cdots \quad(a \in \mathbb{C}, n \in \mathbb{N}=\{1,2, \ldots\}) .
$$

Also, let $A$ be the subclass of $H$ containing of functions of the form:

$$
f(z)=z+\sum_{k=2}^{\infty} a_{k} z^{k} .
$$

Received: September 8, 2019; Accepted: November 13, 2019

2010 Mathematics Subject Classification: 30C45.

Keywords and phrases: analytic function, differential subordination, differential superordination, dominant, subordinant, Frasin operator.

Copyright () 2020 Abbas Kareem Wanas and Sibel Yalçin. This is an open access article distributed under the Creative Commons Attribution License, which permits unrestricted use, distribution, and reproduction in any medium, provided the original work is properly cited. 
Let $f, g \in H$. The function $f$ is said to be subordinate to $g$, or $g$ is said to be superordinate to $f$, if there exists a Schwarz function $w$ analytic in $U$ with $w(0)=0$ and $|w(z)|<1(z \in U)$ such that $f(z)=g(w(z))$. This subordination is denoted by $f \prec g$ or $f(z) \prec g(z)(z \in U)$. It is well known that, if the function $g$ is univalent in $U$, then $f \prec g$ if and only if $f(0)=g(0)$ and $f(U) \subset g(U)$.

Let $\xi, h \in H$ and $\psi(r, s, t ; z): \mathbb{C}^{3} \times U \rightarrow \mathbb{C}$. If $\xi$ and $\psi\left(\xi(z), z \xi^{\prime}(z), z^{2} \xi^{\prime \prime}(z) ; z\right)$ are univalent functions in $U$ and if $\xi$ satisfies the second-order differential superordination

$$
h(z) \prec \psi\left(\xi(z), z \xi^{\prime}(z), z^{2} \xi^{\prime \prime}(z) ; z\right),
$$

then $\xi$ is called a solution of the differential superordination (1.2). (If $f$ is subordinate to $g$, then $g$ is superordinate to $f$ ). An analytic function $q$ is called a subordinant of (1.2), if $q \prec \xi$ for all $\xi$ satisfying (1.2). A univalent subordinant $\tilde{q}$ that satisfies $q \prec \tilde{q}$ for all the subordinants $q$ of (1.2) is called the best subordinant.

For $m \in \mathbb{N}, \quad \lambda, j \in \mathbb{N}_{0}=\mathbb{N} \cup\{0\}, \quad 0 \leq \tau \leq 1$ and $f \in A$, the Frasin operator $D_{m, \tau}^{\lambda}: A \rightarrow A$ (see [4]) is defined by

$$
D_{m, \tau}^{\lambda} f(z)=z+\sum_{k=2}^{\infty}\left(1+(k-1) \sum_{j=1}^{m}\left(\begin{array}{c}
m \\
j
\end{array}\right)(-1)^{j+1} \tau^{j}\right)^{\lambda} a_{k} z^{k}
$$

It is readily verified from (1.3) that

$$
C_{j}^{m}(\tau) z\left(D_{m, \tau}^{\lambda} f(z)\right)_{z}^{\prime}=D_{m, \tau}^{\lambda+1} f(z)-\left(1-C_{j}^{m}(\tau)\right) D_{m, \tau}^{\lambda} f(z)
$$

where $C_{j}^{m}(\tau)=\sum_{j=1}^{m}\left(\begin{array}{c}m \\ j\end{array}\right)(-1)^{j+1} \tau^{j}$

Special cases of this operator include the generalized Sălăgean operator [1], the Sălăgean differential operator [10].

Very recently several authors, Shanmugam et al. [11], Goyal et al. [5], El-Ashwah et al. [3] and Wanas and Majeed [12] have obtained sandwich results for certain classes of analytic functions. 
The main object of the present investigation is to find sufficient conditions for certain normalized analytic functions $f$ to satisfy

$$
q_{1}(z) \prec\left(\frac{D_{m, \tau}^{\lambda} f(z)}{z}\right)^{\gamma} \prec q_{2}(z)
$$

and

$$
q_{1}(z) \prec\left(\frac{D_{m, \tau}^{\lambda+1} f(z)}{D_{m, \tau}^{\lambda} f(z)}\right)^{\gamma} \prec q_{2}(z),
$$

where $q_{1}$ and $q_{2}$ are given univalent functions in $U$ with $q_{1}(0)=q_{2}(0)=1$.

In order to prove our results, we make use of the following known results.

Definition 1.1 [6]. Denote by $Q$ the set of all functions $f$ that are analytic and injective on $\bar{U} \backslash E(f)$, where

$$
E(f)=\left\{\zeta \in \partial U: \lim _{z \rightarrow \zeta} f(z)=\infty\right\}
$$

and are such that $f^{\prime}(\zeta) \neq 0$ for $\zeta \in \partial U \backslash E(f)$.

Lemma 1.1 [6]. Let $q$ be univalent in the unit disk $U$ and let $\theta$ and $\phi$ be analytic in a domain D containing $q(U)$ with $\phi(w) \neq 0$ when $w \in q(U)$. Set $Q(z)=z q^{\prime}(z) \phi(q(z))$ and $h(z)=\theta(q(z))+Q(z)$. Suppose that

(1) $Q(z)$ is starlike univalent in $U$,

(2) $\operatorname{Re}\left\{\frac{z h^{\prime}(z)}{Q(z)}\right\}>0$ for $z \in U$.

If $\xi$ is analytic in $U$, with $\xi(0)=q(0), \xi(U) \subset D$ and

$$
\theta(\xi(z))+z \xi^{\prime}(z) \phi(\xi(z)) \prec \theta(q(z))+z q^{\prime}(z) \phi(q(z)),
$$

then $\xi \prec q$ and $q$ is the best dominant of (1.5).

Lemma 1.2 [7]. Let $q$ be a convex univalent function in $U$ and let $\alpha \in \mathbb{C}, \beta \in \mathbb{C} \backslash\{0\}$ with 


$$
\operatorname{Re}\left\{1+\frac{z q^{\prime \prime}(z)}{q^{\prime}(z)}\right\}>\max \left\{0,-\operatorname{Re}\left(\frac{\alpha}{\beta}\right)\right\}
$$

If $\xi$ is analytic in $U$ and

$$
\alpha \xi(z)+\beta z \xi^{\prime}(z) \prec \alpha q(z)+\beta z q^{\prime}(z),
$$

then $\xi \prec q$ and $q$ is the best dominant of (1.6).

Lemma 1.3 [7]. Let $q$ be convex univalent in $U$ and let $\beta \in \mathbb{C}$. Further assume that $\operatorname{Re}(\beta)>0$. If $\xi \in H[q(0), 1] \cap Q$ and $\xi(z)+\beta z \xi^{\prime}(z)$ is univalent in $U$, then

$$
q(z)+\beta z q^{\prime}(z) \prec \xi(z)+\beta z \xi^{\prime}(z),
$$

which implies that $q \prec \xi$ and $q$ is the best subordinant of (1.7).

Lemma 1.4 [2]. Let $q$ be convex univalent in the unit disk $U$ and let $\theta$ and $\phi$ be analytic in a domain $D$ containing $q(U)$. Suppose that

(1) $\operatorname{Re}\left\{\frac{\theta^{\prime}(q(z))}{\phi(q(z))}\right\}>0$ for $z \in U$,

(2) $Q(z)=z q^{\prime}(z) \phi(q(z))$ is starlike univalent in $U$.

If $\xi \in H[q(0), 1] \cap Q$, with $\xi(U) \subset D, \theta(\xi(z))+z \xi^{\prime}(z) \phi(\xi(z))$ is univalent in $U$ and

$$
\theta(q(z))+z q^{\prime}(z) \phi(q(z)) \prec \theta(\xi(z))+z \xi^{\prime}(z) \phi(\xi(z)),
$$

then $q \prec \xi$ and $q$ is the best subordinant of (1.8).

\section{Main Results}

Theorem 2.1. Let $q$ be convex univalent in $U$ with $q(0)=1, \sigma \in \mathbb{C} /\{0\}, \gamma>0$ and suppose that $q$ satisfies

$$
\operatorname{Re}\left\{1+\frac{z q^{\prime \prime}(z)}{q^{\prime}(z)}\right\}>\max \left\{0,-\operatorname{Re}\left(\frac{\gamma}{\sigma}\right)\right\} .
$$


If $f \in A$ satisfies the subordination

$$
\left(1-\frac{\sigma}{C_{j}^{m}(\tau)}\right)\left(\frac{D_{m, \tau}^{\lambda} f(z)}{z}\right)^{\gamma}+\frac{\sigma}{C_{j}^{m}(\tau)}\left(\frac{D_{m, \tau}^{\lambda} f(z)}{z}\right)^{\gamma}\left(\frac{D_{m, \tau}^{\lambda+1} f(z)}{D_{m, \tau}^{\lambda} f(z)}\right) \prec q(z)+\frac{\sigma}{\gamma} z q^{\prime}(z),
$$

then

$$
\left(\frac{D_{m, \tau}^{\lambda} f(z)}{z}\right)^{\gamma} \prec q(z)
$$

and $q$ is the best dominant of (2.2).

Proof. Define the function $\xi$ by

$$
\xi(z)=\left(\frac{D_{m, \tau}^{\lambda} f(z)}{z}\right)^{\gamma}, \quad(z \in U)
$$

Differentiating (2.4) logarithmically with respect to $z$, we get

$$
\frac{z \xi^{\prime}(z)}{\xi(z)}=\gamma\left(\frac{z\left(D_{m, \tau}^{\lambda} f(z)\right)^{\prime}}{D_{m, \tau}^{\lambda} f(z)}-1\right)
$$

Now, in view of (1.4), we obtain the following subordination

$$
\frac{z \xi^{\prime}(z)}{\xi(z)}=\frac{\gamma}{C_{j}^{m}(\tau)}\left(\frac{D_{m, \tau}^{\lambda+1} f(z)}{D_{m, \tau}^{\lambda} f(z)}-1\right)
$$

Therefore,

$$
\frac{z \xi^{\prime}(z)}{\gamma}=\frac{1}{C_{j}^{m}(\tau)}\left(\frac{D_{m, \tau}^{\lambda} f(z)}{z}\right)^{\gamma}\left(\frac{D_{m, \tau}^{\lambda+1} f(z)}{D_{m, \tau}^{\lambda} f(z)}-1\right) .
$$

The subordination (2.2) from the hypothesis becomes

$$
\xi(z)+\frac{\sigma}{\gamma} z \xi^{\prime}(z) \prec q(z)+\frac{\sigma}{\gamma} z q^{\prime}(z) .
$$

Hence, an application of Lemma 1.2 with $\alpha=1$ and $\beta=\frac{\sigma}{\gamma}$, we obtain (2.3). 
Theorem 2.2. Let $\eta_{i} \in \mathbb{C}(i=1,2,3,4), \quad \gamma>0, \quad \delta \in \mathbb{C} /\{0\}$ and $q$ be convex univalent in $U$ with $q(0)=1, q(z) \neq 0(z \in U)$ and assume that $q$ satisfies

$$
\operatorname{Re}\left\{1+\frac{\eta_{2}}{\delta} q(z)+\frac{2 \eta_{3}}{\delta} q^{2}(z)+\frac{3 \eta_{4}}{\delta} q^{3}(z)+\frac{z q^{\prime \prime}(z)}{q^{\prime}(z)}-\frac{z q^{\prime}(z)}{q(z)}\right\}>0 .
$$

Suppose that $\frac{z q^{\prime}(z)}{q(z)}$ is starlike univalent in $U$. If $f \in A$ satisfies

$$
\begin{aligned}
& \Omega\left(\eta_{1}, \eta_{2}, \eta_{3}, \eta_{4}, \gamma, \delta, \lambda, m, \tau ; z\right) \\
\prec & \eta_{1}+\eta_{2} q(z)+\eta_{3} q^{2}(z)+\eta_{4} q^{3}(z)+\delta \frac{z q^{\prime}(z)}{q(z)},
\end{aligned}
$$

where

$$
\begin{aligned}
& \Omega\left(\eta_{1}, \eta_{2}, \eta_{3}, \eta_{4}, \gamma, \delta, \lambda, m, \tau ; z\right) \\
= & \eta_{1}+\eta_{2}\left(\frac{D_{m, \tau}^{\lambda+1} f(z)}{D_{m, \tau}^{\lambda} f(z)}\right)^{\gamma}+\eta_{3}\left(\frac{D_{m, \tau}^{\lambda+1} f(z)}{D_{m, \tau}^{\lambda} f(z)}\right)^{2 \gamma}+\eta_{4}\left(\frac{D_{m, \tau}^{\lambda+1} f(z)}{D_{m, \tau}^{\lambda} f(z)}\right)^{3 \gamma} \\
& +\frac{\gamma \delta}{C_{j}^{m}(\tau)}\left(\frac{D_{m, \tau}^{\lambda+2} f(z)}{D_{m, \tau}^{\lambda+1} f(z)}-\frac{D_{m, \tau}^{\lambda+1} f(z)}{D_{m, \tau}^{\lambda} f(z)}\right)
\end{aligned}
$$

then

$$
\left(\frac{D_{m, \tau}^{\lambda+1} f(z)}{D_{m, \tau}^{\lambda} f(z)}\right)^{\gamma} \prec q(z)
$$

and $q$ is the best dominant of (2.6).

Proof. Define the function $\xi$ by

$$
\xi(z)=\left(\frac{D_{m, \tau}^{\lambda+1} f(z)}{D_{m, \tau}^{\lambda} f(z)}\right)^{\gamma}, \quad(z \in U)
$$

By a straightforward computation and using (1.4), we have 


$$
\begin{aligned}
& \eta_{1}+\eta_{2} \xi(z)+\eta_{3} \xi^{2}(z)+\eta_{4} \xi^{3}(z)+\delta \frac{z \xi^{\prime}(z)}{\xi(z)} \\
= & \Omega\left(\eta_{1}, \eta_{2}, \eta_{3}, \eta_{4}, \gamma, \delta, \lambda, m, \tau ; z\right),
\end{aligned}
$$

where $\Omega\left(\eta_{1}, \eta_{2}, \eta_{3}, \eta_{4}, \gamma, \delta, \lambda, m, \tau ; z\right)$ is given by (2.7).

From (2.6) and (2.9), we obtain

$$
\begin{gathered}
\eta_{1}+\eta_{2} \xi(z)+\eta_{3} \xi^{2}(z)+\eta_{4} \xi^{3}(z)+\delta \frac{z \xi^{\prime}(z)}{\xi(z)} \\
\prec \eta_{1}+\eta_{2} q(z)+\eta_{3} q^{2}(z)+\eta_{4} q^{3}(z)+\delta \frac{z q^{\prime}(z)}{q(z)} .
\end{gathered}
$$

By setting

$$
\theta(w)=\eta_{1}+\eta_{2} w+\eta_{3} w^{2}+\eta_{4} w^{3} \text { and } \phi(w)=\frac{\delta}{w}, w \neq 0,
$$

we see that $\theta(w)$ is analytic in $\mathbb{C}, \phi(w)$ is analytic in $\mathbb{C} \backslash\{0\}$ and that $\phi(w) \neq 0$, $w \in \mathbb{C} \backslash\{0\}$. Also, we get

$$
Q(z)=z q^{\prime}(z) \phi(q(z))=\delta \frac{z q^{\prime}(z)}{q(z)}
$$

and

$$
h(z)=\theta(q(z))+Q(z)=\eta_{1}+\eta_{2} q(z)+\eta_{3} q^{2}(z)+\eta_{4} q^{3}(z)+\delta \frac{z q^{\prime}(z)}{q(z)} .
$$

It is clear that $Q(z)$ is starlike univalent in $U$,

$$
\operatorname{Re}\left\{\frac{z h^{\prime}(z)}{Q(z)}\right\}=\operatorname{Re}\left\{1+\frac{\eta_{2}}{\delta} q(z)+\frac{2 \eta_{3}}{\delta} q^{2}(z)+\frac{3 \eta_{4}}{\delta} q^{3}(z)+\frac{z q^{\prime \prime}(z)}{q^{\prime}(z)}-\frac{z q^{\prime}(z)}{q(z)}\right\}>0 .
$$

Thus, by Lemma 1.1, we get $\xi(z) \prec q(z)$. By using (2.8), we obtain the desired result.

Theorem 2.3. Let $q$ be convex univalent in $U$ with $q(0)=1, \gamma>0$ and $\operatorname{Re}\{\sigma\}>0$. Let $f \in$ A satisfy 


$$
\left(\frac{D_{m, \tau}^{\lambda} f(z)}{z}\right)^{\gamma} \in H[q(0), 1] \cap Q
$$

and

$$
\left(1-\frac{\sigma}{C_{j}^{m}(\tau)}\right)\left(\frac{D_{m, \tau}^{\lambda} f(z)}{z}\right)^{\gamma}+\frac{\sigma}{C_{j}^{m}(\tau)}\left(\frac{D_{m, \tau}^{\lambda} f(z)}{z}\right)^{\gamma}\left(\frac{D_{m, \tau}^{\lambda+1} f(z)}{D_{m, \tau}^{\lambda} f(z)}\right)
$$

be univalent in $U$. If

$$
\begin{aligned}
& q(z)+\frac{\sigma}{\gamma} z q^{\prime}(z) \\
\prec & \left(1-\frac{\sigma}{C_{j}^{m}(\tau)}\right)\left(\frac{D_{m, \tau}^{\lambda} f(z)}{z}\right)^{\gamma}+\frac{\sigma}{C_{j}^{m}(\tau)}\left(\frac{D_{m, \tau}^{\lambda} f(z)}{z}\right)^{\gamma}\left(\frac{D_{m, \tau}^{\lambda+1} f(z)}{D_{m, \tau}^{\lambda} f(z)}\right),
\end{aligned}
$$

then

$$
q(z) \prec\left(\frac{D_{m, \tau}^{\lambda} f(z)}{z}\right)^{\gamma}
$$

and $q$ is the best subordinant of (2.10).

Proof. Let the function $\xi$ be defined by (2.4). After some computations and using (1.4), it is evident that

$$
\begin{aligned}
& \left(1-\frac{\sigma}{C_{j}^{m}(\tau)}\right)\left(\frac{D_{m, \tau}^{\lambda} f(z)}{z}\right)^{\gamma}+\frac{\sigma}{C_{j}^{m}(\tau)}\left(\frac{D_{m, \tau}^{\lambda} f(z)}{z}\right)^{\gamma}\left(\frac{D_{m, \tau}^{\lambda+1} f(z)}{D_{m, \tau}^{\lambda} f(z)}\right) \\
= & \xi(z)+\frac{\sigma}{\gamma} z \xi^{\prime}(z) .
\end{aligned}
$$

From (2.10) and (2.12), we get

$$
q(z)+\frac{\sigma}{\gamma} z q^{\prime}(z) \prec \xi(z)+\frac{\sigma}{\gamma} z \xi^{\prime}(z)
$$

Hence, an application of Lemma 1.3 with $\alpha=1$ and $\beta=\frac{\sigma}{\gamma}$, we obtain (2.11). 
Theorem 2.4. Let $\eta_{i} \in \mathbb{C}(i=1,2,3,4), \gamma>0, \delta \in \mathbb{C} /\{0\}$ and $q$ be convex univalent in $U$ with $q(0)=1, q(z) \neq 0(z \in U)$ and assume that $q$ satisfies

$$
\operatorname{Re}\left\{\frac{\eta_{2}}{\delta} q(z)+\frac{2 \eta_{3}}{\delta} q^{2}(z)+\frac{3 \eta_{4}}{\delta} q^{3}(z)\right\}>0
$$

Suppose that $\frac{z q^{\prime}(z)}{q(z)}$ is starlike univalent in U. Let $f \in A$ satisfy

$$
\left(\frac{D_{m, \tau}^{\lambda+1} f(z)}{D_{m, \tau}^{\lambda} f(z)}\right)^{\gamma} \in H[q(0), 1] \cap Q
$$

and $\Omega\left(\eta_{1}, \eta_{2}, \eta_{3}, \eta_{4}, \gamma, \delta, \lambda, m, \tau ; z\right)$ is univalent in $U$, where $\Omega\left(\eta_{1}, \eta_{2}, \eta_{3}, \eta_{4}\right.$, $\gamma, \delta, \lambda, m, \tau ; z)$ is given by (2.7). If

$$
\begin{aligned}
& \eta_{1}+\eta_{2} q(z)+\eta_{3} q^{2}(z)+\eta_{4} q^{3}(z)+\delta \frac{z q^{\prime}(z)}{q(z)} \\
\prec & \Omega\left(\eta_{1}, \eta_{2}, \eta_{3}, \eta_{4}, \gamma, \delta, \lambda, m, \tau ; z\right),
\end{aligned}
$$

then

$$
q(z) \prec\left(\frac{D_{m, \tau}^{\lambda+1} f(z)}{D_{m, \tau}^{\lambda} f(z)}\right)^{\gamma}
$$

and $q$ is the best subordinant of (2.14).

Proof. Let the function $\xi$ be defined by (2.8). By a straightforward computation and using (1.4), we find that

$$
\begin{aligned}
& \Omega\left(\eta_{1}, \eta_{2}, \eta_{3}, \eta_{4}, \gamma, \delta, \lambda, m, \tau ; z\right) \\
= & \eta_{1}+\eta_{2} \xi(z)+\eta_{3} \xi^{2}(z)+\eta_{4} \xi^{3}(z)+\delta \frac{z \xi^{\prime}(z)}{\xi(z)},
\end{aligned}
$$

where $\Omega\left(\eta_{1}, \eta_{2}, \eta_{3}, \eta_{4}, \gamma, \delta, \lambda, m, \tau ; z\right)$ is given by (2.7).

From (2.14) and (2.15), we obtain

$$
\eta_{1}+\eta_{2} q(z)+\eta_{3} q^{2}(z)+\eta_{4} q^{3}(z)+\delta \frac{z q^{\prime}(z)}{q(z)}
$$




$$
\prec \eta_{1}+\eta_{2} \xi(z)+\eta_{3} \xi^{2}(z)+\eta_{4} \xi^{3}(z)+\delta \frac{z \xi^{\prime}(z)}{\xi(z)} .
$$

By setting $\theta(w)=\eta_{1}+\eta_{2} w+\eta_{3} w^{2}+\eta_{4} w^{3}$ and $\phi(w)=\frac{\delta}{w}, w \neq 0$, we see that $\theta(w)$ is analytic in $\mathbb{C}, \phi(w)$ is analytic in $\mathbb{C} \backslash\{0\}$ and that $\phi(w) \neq 0, w \in \mathbb{C} \backslash\{0\}$. Also, we get

$$
Q(z)=z q^{\prime}(z) \phi(q(z))=\delta \frac{z q^{\prime}(z)}{q(z)} .
$$

It is clear that $Q(z)$ is starlike univalent in $U$,

$$
\operatorname{Re}\left\{\frac{\theta^{\prime}(q(z))}{\phi(q(z))}\right\}=\operatorname{Re}\left\{\frac{\eta_{2}}{\delta} q(z)+\frac{2 \eta_{3}}{\delta} q^{2}(z)+\frac{3 \eta_{4}}{\delta} q^{3}(z)\right\}>0 .
$$

Thus, by Lemma 1.4, we get $q(z) \prec \xi(z)$. By using (2.8), we obtain the desired result.

Concluding the results of differential subordination and superordination, we state the following "sandwich results".

Theorem 2.5. Let $q_{1}$ and $q_{2}$ be convex univalent in $U$ with $q_{1}(0)=q_{2}(0)=1$. Suppose $q_{2}$ satisfies (2.1), $\gamma>0$ and $\operatorname{Re}\{\sigma\}>0$. Let $f \in A$ satisfy

$$
\left(\frac{D_{m, \tau}^{\lambda} f(z)}{z}\right)^{\gamma} \in H[1,1] \cap Q
$$

and

$$
\left(1-\frac{\sigma}{C_{j}^{m}(\tau)}\right)\left(\frac{D_{m, \tau}^{\lambda} f(z)}{z}\right)^{\gamma}+\frac{\sigma}{C_{j}^{m}(\tau)}\left(\frac{D_{m, \tau}^{\lambda} f(z)}{z}\right)^{\gamma}\left(\frac{D_{m, \tau}^{\lambda+1} f(z)}{D_{m, \tau}^{\lambda} f(z)}\right)
$$

be univalent in $U$. If

$$
\begin{aligned}
& q_{1}(z)+\frac{\sigma}{\gamma} z q_{1}^{\prime}(z) \\
\prec & \left(1-\frac{\sigma}{C_{j}^{m}(\tau)}\right)\left(\frac{D_{m, \tau}^{\lambda} f(z)}{z}\right)^{\gamma}+\frac{\sigma}{C_{j}^{m}(\tau)}\left(\frac{D_{m, \tau}^{\lambda} f(z)}{z}\right)^{\gamma}\left(\frac{D_{m, \tau}^{\lambda+1} f(z)}{D_{m, \tau}^{\lambda} f(z)}\right)
\end{aligned}
$$




$$
\prec q_{2}(z)+\frac{\sigma}{\gamma} z q_{2}^{\prime}(z)
$$

then

$$
q_{1}(z) \prec\left(\frac{D_{m, \tau}^{\lambda} f(z)}{z}\right)^{\gamma} \prec q_{2}(z)
$$

and $q_{1}$ and $q_{2}$ are, respectively, the best subordinant and the best dominant.

Theorem 2.6. Let $q_{1}$ and $q_{2}$ be convex univalent in $U$ with $q_{1}(0)=q_{2}(0)=1$. Suppose $q_{1}$ satisfies (2.13) and $q_{2}$ satisfies (2.5). Let $f \in A$ satisfies

$$
\left(\frac{D_{m, \tau}^{\lambda+1} f(z)}{D_{m, \tau}^{\lambda} f(z)}\right)^{\gamma} \in H[1,1] \cap Q
$$

and $\Omega\left(\eta_{1}, \eta_{2}, \eta_{3}, \eta_{4}, \gamma, \delta, \lambda, m, \tau ; z\right)$ is univalent in $U$, where $\Omega\left(\eta_{1}, \eta_{2}, \eta_{3}, \eta_{4}\right.$, $\gamma, \delta, \lambda, m, \tau ; z)$ is given by (2.7). If

$$
\begin{aligned}
& \eta_{1}+\eta_{2} q_{1}(z)+\eta_{3} q_{1}^{2}(z)+\eta_{4} q_{1}^{3}(z)+\delta \frac{z q_{1}^{\prime}(z)}{q_{1}(z)} \\
\prec & \Omega\left(\eta_{1}, \eta_{2}, \eta_{3}, \eta_{4}, \gamma, \delta, \lambda, m, \tau ; z\right) \\
\prec & \eta_{1}+\eta_{2} q_{2}(z)+\eta_{3} q_{2}^{2}(z)+\eta_{4} q_{2}^{3}(z)+\delta \frac{z q_{2}^{\prime}(z)}{q_{2}(z)},
\end{aligned}
$$

then

$$
q_{1}(z) \prec\left(\frac{D_{m, \tau}^{\lambda+1} f(z)}{D_{m, \tau}^{\lambda} f(z)}\right)^{\gamma} \prec q_{2}(z)
$$

and $q_{1}$ and $q_{2}$ are, respectively, the best subordinant and the best dominant.

Remark 2.1. By selecting the particular values of $m, \tau$ and $\lambda$, we can derive a number of known results. Some of them are given below.

(1) Taking $m=1$ in Theorems 2.1, 2.3 and 2.5, we have the results obtained by Răducanu and Nechita [9, Theorem 3.1, Theorem 3.6, Theorem 3.9]. 
(2) Putting $m=\tau=1$ in Theorems 2.1, 2.3 and 2.5, we get the results obtained by Răducanu and Nechita [9, Corollary 3.3, Corollary 3.8, Corollary 3.11].

(3) Setting $m=\tau=1$ and $\lambda=1$ in Theorem 2.1, we obtain the results obtained by Murugusundaramoorthy and Magesh [8, Corollary 3.3].

(4) Taking $m=\tau=1$ and $\lambda=1$ in Theorems 2.3 and 2.5, we have the results obtained by Răducanu and Nechita [9, Corollary 3.7, Corollary 3.10].

\section{References}

[1] F. M. Al-Oboudi, On univalent functions defined by a generalized Sălăgean operator, Int. J. Math. Math. Sci. 2004, no. 25-28, 1429-1436. https://doi.org/10.1155/S0161171204108090

[2] T. Bulboacă, Classes of first-order differential superordinations, Demonstratio Math. 35(2) (2002), 287-292. https://doi.org/10.1515/dema-2002-0209

[3] R. M. El-Ashwah, M. K. Aouf, A. Shamandy and S. M. El-Deeb, Differential sandwich theorems of symmetric points associated with Dziok-Srivastava operator, Matematiche (Catania) 68(2) (2013), 249-266.

[4] B. Frasin, A new differential operator of analytic functions involving binomial series, Bol. Soc. Paran. Mat. 38(5) (2020), 205-213. https://doi.org/10.5269/bspm.v38i5.40188

[5] S. P. Goyal, P. Goswami and H. Silverman, Subordination and superordination results for a class of analytic multivalent functions, Int. J. Math. Math. Sci. 2008, Art. ID 561638, 12 pp. https://doi.org/10.1155/2008/561638

[6] S. S. Miller and P. T. Mocanu, Differential subordinations: theory and applications, Monographs and Textbooks in Pure and Applied Mathematics, 225, Marcel Dekker, Inc., New York and Basel, 2000.

[7] S. S. Miller and P. T. Mocanu, Subordinants of differential superordinations, Complex Variables 48(10) (2003), 815-826. https://doi.org/10.1080/02781070310001599322

[8] G. Murugusundaramoorthy and N. Magesh, Differential subordinations and superordinations for analytic functions defined by Dziok-Srivastava linear operator, JIPAM. J. Inequal. Pure Appl. Math. 7(4) (2006), Art. 152, 9 pp.

[9] D. Răducanu and V. O. Nechita, A differential sandwich theorem for analytic functions defined by the generalized Sălăgean operator, Aust. J. Math. Anal. Appl. 9(1) (2012), Art. $8,7 \mathrm{pp}$. 
[10] G. S. Sălăgean, Subclasses of univalent functions, Lecture Notes in Math., 1013, Springer Verlag, Berlin, 1983, pp. 362-372. https://doi.org/10.1007/BFb0066543

[11] T. N. Shanmugam, V. Ravichandran and S. Sivasubramanian, Differential sandwich theorems for some subclasses of analytic functions, Aust. J. Math. Anal. Appl. 3(1) (2006), Art. 8, 11 pp.

[12] A. K. Wanas and A. H. Majeed, Differential sandwich theorems for multivalent analytic functions defined by convolution structure with generalized hypergeometric function, $A n$. Univ. Oradea Fasc. Math. 25(2) (2018), 37-52. 